\title{
A retrospective study to evaluate the time burden associated with outpatient red blood transfusions indicated for anemia due to concomitantly administered chemotherapy in cancer patients
}

\author{
Sanatan Shreay • Marie-Pierre Desrosiers • \\ Patricia Corey-Lisle • Krista Payne
}

Received: 12 April 2012 / Accepted: 19 November 2012 /Published online: 22 December 2012

(C) The Author(s) 2012. This article is published with open access at Springerlink.com

\begin{abstract}
Purpose Anemia in cancer patients can be treated with red blood cell (RBC) transfusions. The patient burden associated with a treatment in terms of total time spent is an important factor to consider when measuring the benefits and challenges of a therapy. This study estimates the time-related patient burden associated with outpatient RBC transfusion.

Methods A retrospective chart review of outpatient cancer patients receiving a $\mathrm{RBC}$ transfusion was conducted at 10 US centers. RBC transfusion time was measured as time elapsed from pre- to post-transfusion vital sign assessment and from transfusion start to stop time. Elapsed time from hemoglobin level testing and blood draw for cross-match to transfusion, estimated travel time and distance, and clinical and demographic data were also collected.

Results Data from 110 patients (48.2 \% male; mean age $64 \pm$ 12 years) showed that the mean elapsed time between pre- and post-vital sign assessment was $4.2 \mathrm{~h}(95 \%$ confidence interval (CI), 3.64-4.81) including $3.6 \mathrm{~h}$ (95\% CI, 3.0-4.1) on average to receive the actual RBC transfusion treatment. Hemoglobin level testing (mean $\mathrm{Hg}$ level, $8.33 \mathrm{~g} / \mathrm{dL} \pm 0.67$ ) and blood drawn for cross-match were completed in an average of $31.2 \mathrm{~h}(95 \% \mathrm{CI}, 17.0-45.5)$ and $18.2 \mathrm{~h}(95 \% \mathrm{CI}, 12.1-$ $24.2)$ prior to transfusion, respectively. Patient one-way travel time averaged $30.0 \mathrm{~min}$ (95\% CI, 25.9-34.3).

Conclusions In the US, CIA patients experience an important time burden when being treated with RBC transfusion in addition to the burden already added by chemotherapy.
\end{abstract}

S. Shreay $\cdot$ P. Corey-Lisle

Amgen Inc, Thousand Oaks, CA, USA

M.-P. Desrosiers $(\bowtie) \cdot K$. Payne

United BioSource Corporation, Montreal, QC, Canada

e-mail: marie-pierre.desrosiers@unitedbiosource.com
Keywords Anemia - Chemotherapy $\cdot$ RBC transfusion . Patient burden

\section{Introduction}

Anemia is defined as a deficiency in the concentration of hemoglobin containing red blood cells (RBCs). Anemia symptoms including fatigue, dizziness, shortness of breath, palpitations and cardiovascular complications, depression, and impaired immune function can negatively impact patient functional capacity and worsen patient health-related quality of life $[1,2]$. Anemia incidence can be as high as $90 \%$ in cancer patients receiving chemotherapy, radiation therapy, or both [3-5]. Anemia is a common complication in cancer patients receiving concomitantly administered chemotherapy (subsequently called chemotherapy-induced anemia; CIA) [3]. CIA results from direct effects of chemotherapy on bone marrow function and renal function, which impair the production of RBC [3]. Standard treatments for CIA patients include $\mathrm{RBC}$ transfusion, iron therapy, and medication to stimulate formation of RBC [4].

Multiple publications provide an assessment of the time burden associated with receiving erythropoietin-stimulating agents (ESA) therapy (specifically epoetin alpha and darbepoetin alfa $[6,7]$ ) and only certain studies reported $\mathrm{RBC}$ transfusion time burden from site perspective. In fact, one single study conducted in Canada prospectively examined the "chair time" for 44 RBC transfusions in 38 patients while another single-center study retrospective chart review in the US assessed the transfusion time for 100 transfusions among 36 patients (19 patients with CIA) [8, 9]. Although these studies estimate the time required for outpatient 
transfusion, these published studies estimate the time burden from a site perspective and they are produced from singlecenter studies. No research studies specifically aimed at estimating the patient time burden of RBC transfusion to treat CIA in multiple centers have been identified.

This study was undertaken to estimate the patient burden in terms of time spent that is associated with outpatient RBC transfusion treatment indicated for anemia caused by concomitantly administered chemotherapy in patients with non-myeloid malignancies.

\section{Methods}

A retrospective chart review study was implemented to collect and summarize patient data reflecting patient burden in terms of the time spent associated with outpatient RBC transfusions indicated for CIA in patients with non-myeloid malignancies.

\section{Study design}

This study is a multi-center, cross-sectional, retrospective chart review study that was conducted at 10 US sites. Transfusion centers that administer RBC transfusion indicated for CIA in the outpatient setting that routinely document the time the patients spend at the site in pre-transfusion and posttransfusion vital signs assessment activities were considered for participation in this study. In addition, potential sites underwent a feasibility assessment in which site enrollment eligibility criteria including size of patient population and availability of patient medical records were evaluated.

At each participating center, patients with a diagnosis of non-myeloid malignancy undergoing $\mathrm{RBC}$ transfusion to treat their CIA were identified, screened, and recruited to be included in this study. Eligible patients' medical records that document patient and treatment characteristics and time spent in clinic during a patient's transfusion visit were used to quantify the outpatient RBC transfusion treatment time. Anonymized data from the patient's medical records was abstracted by local site study staff, recorded on paper case report forms (CRFs), and entered at the site into an electronic data capture (EDC) system. The study protocol was approved by the appropriate IRB in the USA and waiver of patient consent was obtained. Also, this study was conducted according to the recommendations of the Declaration of Helsinki [10].

Using a site-specific chart identification algorithm, study sites identified all patients with a diagnosis of non-myeloid malignancy who received an outpatient RBC transfusion indicated for CIA within a 3-month eligibility period from August 01, 2010 to October 31, 2010. Each patient identified was entered into an anonymized electronic enrollment log, assigned with a study identification number and uploaded to a secure, password-protected EDC system designed and managed by the Study Coordinating Center (United BioSource Corporation, Lexington, USA). All patients identified were randomly selected using the EDC system, and a preliminary cohort of patients was identified. Only the first eligible RBC transfusion visit per patient was considered. In the order of the EDC system randomization, the site reviewed the chart of the patients listed in the preliminary target cohort to confirm study eligibility using the screening form. To be included in the study, patients were required to have a diagnosis of non-myeloid malignancy (including all solid tumors, non-Hodgkin's lymphoma, Hodgkin's lymphoma, chronic lymphocytic leukemia, and multiple myeloma), have received a RBC transfusion indicated for CIA (as documented in the patient medical chart) that was initially planned to occur in the outpatient setting, and be 18 years of age or greater at time of RBC transfusion. Patients with a diagnosis of acute leukemias, chronic myelogenous leukemia, myelodysplastic syndromes, or marrow fibrosis were excluded from the study. Also, given that study data were collected from the patient's medical chart, electronic or paper medical record of the patient needed to be available for review and abstraction by the study site staff. Finally, patients were excluded if they had received other therapies besides their RBC transfusion during the same visit as the $\mathrm{RBC}$ transfusion visit (excluding diphenhydramine [Benadryl], corticosteroids, ESA, or acetaminophen within the time the patient arrived for their transfusion and the time they left the facility). Patients were screened for eligibility until a total target cohort of approximately 11 eligible patients were identified at the site, for a total of 110 patients among all sites.

\section{Data collection and study endpoints}

For all eligible patients, demographic and clinical data as well as the date and time of pre-determined activities in relation to a patient's transfusion visit was collected from the patient's medical chart, recorded on paper CRF, and entered into EDC system by site study staff. Site study staff also had to complete a site questionnaire regarding study site location, geographic setting, facility type, profit status, teaching status, as well as number of outpatient transfusion beds/chairs at the site and number of transfusions that can be performed at one time at the site.

The primary endpoints for this study are related to the time spent by patients undergoing RBC transfusion treatment measured by the time from pre-transfusion vital signs assessment to post-transfusion vital signs assessment at the transfusion center as well as the outpatient RBC transfusion time (transfusion start time and transfusion stop time).

The secondary endpoints for this study include the elapsed time (in hours) from pre-transfusion hemoglobin level testing 
to transfusion, calculated from date and time of hemoglobin level testing and date and time of start of RBC transfusion; elapsed time (in hours) from blood draw for cross-match to transfusion, calculated from date and time of blood draw for cross-match and date and time of start of RBC transfusion; number of RBC transfusion visits over the course of the most recent year; time spent traveling (in minutes) and distance traveled (in miles) between transfusion center and the patient's residence on the day of the $\mathrm{RBC}$ transfusion visit. MapQuest mapping software [11] and patient address information were used to calculate the time and distance traveled. To be conservative, with respect to the time component of the travel burden, the fastest route was assumed for purposes of calculating distance and time for all patients.

The following exploratory endpoints for secondary objectives were also considered: pre-transfusion hemoglobin levels (level at time oncologist's transfusion order was made, if available) and location, site of blood drawn for cross-match, and number of blood units ordered and transfused. Our study also aimed to explore transfusion reactions and hospitalization during or after the RBC transfusion. However, no transfusion reactions were reported and hospitalization was only reported for one patient in our study.

\section{Statistical analyses}

All enrolled patients were included in the analysis. The unit of analysis for this study is the patient; only one RBC transfusion visit was included per patient. The analyses of the patient demographic and clinical characteristics (specifically, age, gender, race, and type of malignancy) and primary and secondary endpoints are descriptive of patient time burden associated with RBC transfusion indicated for CIA. The analyses for these primary, secondary and exploratory endpoints were descriptive summary methods including mean, median, standard deviation, and range for continuous variables and tables with percentages for categorical variables. Where appropriate, $95 \%$ confidence intervals (CI) for the mean level over all patients were also completed.

\section{Results}

\section{Patient population}

A total of 380 patients with a diagnosis of non-myeloid malignancy who received an outpatient $\mathrm{RBC}$ transfusion indicated for CIA were identified within the 3-month eligibility period from August 01, 2010 to October 31, 2010. From these patients, a total of 186 patients were randomly selected and screened for participation in the study; a total 110 patients met all the study eligibility criteria and therefore were found eligible and included in the final study cohort (mean number of 11 patients per site; minimum 6 patients, maximum 19 patients).

Demographic and clinical characteristics of the final study cohort are summarized in Table 1 . The mean age was $64 \pm 12$ years (range $28-84$ years). About $48 \%$ of the study participants were male, with $74.5 \%$ being white/ Caucasian, $16.4 \%$ Hispanic or Latino/Latin American, and $9.1 \%$ African American. The most common cancer diagnoses were lung cancer $(24.5 \%)$, non-Hodgkin lymphoma (20.9\%), breast cancer (10.9\%), and gastrointestinal/colorectal cancer $(9.1 \%)$. Data on pre-transfusion hemoglobin level was reported for 108 patients (98\%). Among those patients, 29 (26.4\%) patients had hemoglobin level below $8 \mathrm{~g} / \mathrm{dL}$ before transfusion, 73 (66.4\%) patients had hemoglobin level between 8.0 and $9.5 \mathrm{~g} / \mathrm{dL}$, and 6 (5.5\%) patients had hemoglobin level between 9.5 and $13 \mathrm{~g} / \mathrm{dL}$ (mean hemoglobin level, $8.33 \mathrm{~g} / \mathrm{dL} \pm 0.67$ ). Among all patients, a total of 216 blood units were ordered while a total of 211 blood units were transfused (difference of 5 blood units). A total of 17 (15.5\%) patients were ordered 1 unit, 81 (73.6\%) were ordered 2 units, 11 (10.0\%) were ordered 3 units, and only 1 patient $(0.9 \%)$ were ordered at least 4 units. A total of $20(18.2 \%)$ patients had 1 unit transfused, $80(72.7 \%)$ patients had 2 units transfused, 9 $(8.2 \%)$ had 3 units transfused, and only 1 patient $(0.9 \%)$ had at least 4 units transfused.

\section{Study sites}

A total of 10 US sites from different states (Kentucky, Ohio, California, Connecticut, Louisiana, Maryland, Texas, and Alabama) were recruited to participate in the study. Of the 10 participating sites, six sites are outpatient hospitals and four are oncologist office/private practice. Most of the sites (six sites) are located in urban area while two sites are located in suburban area and two sites in rural area. The majority of the sites (seven sites) are considered profit organization and six sites are considered as teaching centers. Among all sites, the mean number of outpatient transfusion beds/ chairs at the site was $14 \pm 18$ beds/chairs and the maximum number of transfusions that can be performed at one time at the site was $7 \pm 6$ transfusions.

Patient time burden

This study collected data to assess the patient time burden associated with the RBC transfusion treatment as well as with clinical tests being performed pre-RBC transfusion treatment and with travel to the clinic to receive $\mathrm{RBC}$ transfusion. The duration of RBC transfusion as well as the time intervals between transfusion-related activities are presented in Table 2. 
Table 1 Demographic and clinical characteristics of study cohort
${ }^{\mathrm{a}}$ Data on type of malignancy was reported to be missing from the medical chart for one patient

\begin{tabular}{|c|c|c|c|}
\hline \multicolumn{2}{|l|}{ Characteristics } & \multicolumn{2}{|l|}{ Characteristics } \\
\hline All patients (\%) & $110(100 \%)$ & All patients $(\%)$ & $110(100 \%)$ \\
\hline Age (years) & & $\mathrm{Hb}$ level, $n(\%)$ & \\
\hline Mean \pm SD & $64 \pm 12$ & Below $8 \mathrm{~g} / \mathrm{dL}$ & $29(26.4 \%)$ \\
\hline Median & 67 & $8.0-9.5 \mathrm{~g} / \mathrm{dL}$ & $73(66.4 \%)$ \\
\hline Range & $28-84$ & $>9.5-13 \mathrm{~g} / \mathrm{dL}$ & $6(5.5 \%)$ \\
\hline Gender, $n(\%)$ & & Hg level unknown & $2(2 \%)$ \\
\hline Male, $n(\%)$ & $53(48.2 \%)$ & Blood units ordered, $n(\%)$ & \\
\hline Female, $n(\%)$ & $57(51.8 \%)$ & 1 unit & $17(15.5 \%)$ \\
\hline Race, $n(\%)$ & & 2 units & $81(73.6 \%)$ \\
\hline $\begin{array}{l}\text { White/Caucasian including } \\
\text { Arab/Middle Eastern }\end{array}$ & $82(74.5 \%)$ & 3 units & $11(10.0 \%)$ \\
\hline Hispanic or Latino/Latin American & $18(16.4 \%)$ & 4+units & $1(0.9 \%)$ \\
\hline Black or African American & $10(9.1 \%)$ & Blood units transfused, $n(\%)$ & \\
\hline Malignancy, $n(\%)$ & & 1 unit & $20(18.2 \%)$ \\
\hline Breast & $12(10.9 \%)$ & 2 units & $80(72.7 \%)$ \\
\hline Head/neck & $3(2.7 \%)$ & 3 units & $9(8.2 \%)$ \\
\hline Gastrointestinal/colorectal & $10(9.1 \%)$ & 4+units & $1(0.9 \%)$ \\
\hline Lung & $27(24.5 \%)$ & & \\
\hline Gynecological & $9(8.2 \%)$ & & \\
\hline Urological & $3(2.7 \%)$ & & \\
\hline Non-Hodgkin's lymphoma & $23(20.9 \%)$ & & \\
\hline Multiple myeloma & $7(6.4 \%)$ & & \\
\hline Chronic lymphocytic leukemia & $2(1.8 \%)$ & & \\
\hline Other & $13(11.8 \%)$ & & \\
\hline Unknown $^{\mathrm{a}}$ & $1(0.9 \%)$ & & \\
\hline
\end{tabular}

\section{$R B C$ transfusion treatment}

The patient direct time burden associated with RBC transfusion treatment was measured by collecting the time from pre-transfusion vital signs assessment and time for posttransfusion vital signs assessment. Time for pre- and posttransfusion vital signs assessments were reported for a total of 98 patients ( $89 \%$ ). For those patients, the mean elapsed time between pre-transfusion vital signs assessment to posttransfusion vital signs assessment is $4.2 \mathrm{~h}(95 \% \mathrm{CI}$, 3.64$4.81)$, including $3.6 \mathrm{~h}(95 \% \mathrm{CI}, 3.0-4.1)$ in average to receive the actual $\mathrm{RBC}$ transfusion treatment. Considering that patients had a mean $( \pm \mathrm{SD})$ of $1.9 \pm 0.5$ blood units transfused, this suggests that patients spent $1.9 \mathrm{~h}$ in average per blood unit transfused per transfusion. The number of $\mathrm{RBC}$ transfusions experienced over the course of the most recent year was reported for 100 patients $(91 \%)$. In this study cohort, $42.0 \%$ of patients had one transfusion over the most recent year, $16.0 \%$ had two transfusions, $28.0 \%$ had three to five transfusions, and $14.0 \%$ patients had more than five transfusions. Considering that the median number of $\mathrm{RBC}$ transfusion experienced during the most recent year was
Table 2 Patient RBC transfusion time burden

\begin{tabular}{lcccc}
\hline Pre-RBC transfusion visit time (hours) & Mean & SE & Range & $95 \%$ CI \\
\hline Hemoglobin level testing to transfusion & 31.2 & 6.3 & $0.88-120.8$ & $17.0-45.5$ \\
Blood draw for cross-match to transfusion & 18.2 & 2.6 & $0.88-67.3$ & $12.1-24.2$ \\
RBC transfusion elapsed time (hours) & & & & \\
Pre- to post-vital signs assessment time & 4.2 & 0.2 & $1.75-6.9$ & $3.6-4.8$ \\
RBC transfusion start time to stop time & 3.6 & 0.2 & $1.1-6.4$ & $3.0-4.1$ \\
Travel burden & & & & \\
Distance (miles) & 21.5 & 2.7 & $0.8-120.0$ & $14.9-28.2$ \\
Time (hours) & 30.0 & 2.1 & $2.0-140.0$ & $25.9-34.0$ \\
\hline
\end{tabular}


2.0 and that patients spent $3.6 \mathrm{~h}$ in average to receive their actual RBC transfusion, this represents up to a total of $7.2 \mathrm{~h}$ per year spent in relation to the administration of RBC transfusion only.

\section{Pre-RBC transfusion treatment}

Time of the pre-transfusion hemoglobin testing and time of start of transfusion was reported for a total of 98 patients (89\%). The mean elapsed time between pre-transfusion hemoglobin test and start of RBC transfusion is $31.24 \mathrm{~h}$ (95\% CI, 17.0-45.5). The results indicate that patients had to wait from 0.88 to $120.80 \mathrm{~h}$ (median hours, 22.36) between pre-transfusion hemoglobin test and start of RBC transfusion. From the total number of patients, $38(34.5 \%)$ patients went to an outpatient hospital to have their hemoglobin level test performed while 68 $(61.8 \%)$ patients went to an oncologist office/private practice setting and only one $(0.9 \%)$ patient went to a transfusion center laboratory (data on site location not reported for one patient).

Time of the blood draw for cross-match and time of start of RBC transfusion was reported for a total of 81 patients $(73.6 \%)$. The mean elapsed time between blood draw for cross-match and start of RBC transfusion is $18.2 \mathrm{~h}(95 \% \mathrm{CI}$, 12.1-24.2). The results indicate that patients had to wait from 0.88 to $67.33 \mathrm{~h}$ (median $20.4 \mathrm{~h}$ ) between blood draw for cross-match and start of RBC transfusion. To be noted is that this time overlaps with the elapse time between hemoglobin test and start of RBC transfusion. Among all patients, $79(71.8 \%)$ patients went to an outpatient hospital setting for their blood drawn for cross-match, 22 (20.0\%) patients went to an oncologist office/private practice setting, and 9 $(8.2 \%)$ patients went to a transfusion center laboratory setting.

\section{Patient travel}

The patient's one-way travel to outpatient center during $\mathrm{RBC}$ transfusion day was also considered to be a factor on patient time burden. Data on distance traveled was reported for a total of 102 patients $(93 \%)$ while data on travel time was reported for all patients. The results show that patients have to travel for a mean distance of 21.5 miles $(95 \% \mathrm{CI}$, 14.9-28.2) from their residence to the RBC transfusion center on the day of the RBC transfusion visit. The largest category of study patients $(27.5 \%)$ traveled between 15 and 25 miles, and $19.6 \%$ had to travel between 25 and 50 miles. The mean traveling time to transfusion center is $30.0 \mathrm{~min}$ (95\% CI, 25.9-34.3). The largest category of study patients $(33.6 \%)$ traveled between 25 and 50 min between the transfusion center and their residence on the day of the $\mathrm{RBC}$ transfusion visit.

\section{Discussion}

To our knowledge, this is the first multi-center study documenting the patient time burden of RBC transfusions to treat CIA. Results from this study on 110 patients demonstrated that patients had to spend $4.2 \mathrm{~h}(95 \% \mathrm{CI}, 3.64-4.81)$ between pre- and post-vital sign assessment, including $3.6 \mathrm{~h}$ to receive their actual RBC transfusion. Two published studies estimating burden from a site perspective have reported patient time for $\mathrm{RBC}$ transfusion $[8,9]$. One single-center study conducted in Canada prospectively examined "chair time" (defined as the time difference between the admission of each patient into care to their time of discharge) through time and motion methodology for 44 RBC transfusion visits. The reported average chair time was $231 \mathrm{~min}( \pm 47 \mathrm{~min})$ with an average of 2.2 units transfused (corresponding to mean chair time per unit of blood of $109 \pm 19 \mathrm{~min}$ ) [8]. The retrospective chart review singlecenter study by Ueno et al. [9] conducted a study including 20 CIA patients and reported a mean transfusion duration of $103( \pm 18.6)$ and $102( \pm 18.3)$ min for the first and second units respectively and for the 2-unit visits, a mean duration between units of $18( \pm 10.0) \mathrm{min}$. Thus, the total average transfusion time reported time for a 2-unit transfusion (time from first unit through the end of the second unit) was $223 \mathrm{~min}( \pm 54)$ or $3.7 \mathrm{~h}$, essentially similar to the transfusion time of $3.6 \mathrm{~h}$ (for 2.0 units in average) we are reporting here.

While there are estimates of patient time required for outpatient transfusion visits, these estimates are limited strictly to the procedure time and do not account for other clinical tests, multiple visits, and travel to site. Our study has taken into account the elapsed time between hemoglobin testing and blood draw for cross-match and RBC transfusion, tests that were completed in an average of $31.24 \mathrm{~h}$ (95\% CI, 17.0-45.5) and $18.20 \mathrm{~h}(95 \% \mathrm{CI}, 17.0-45.5)$ prior to transfusion, respectively. These results demonstrate patient waiting time after their blood tests to receive their RBC transfusion. Although this study demonstrates such delay, it does not allow identifying the potential delay factors such as delay for the $\mathrm{Hb}$ level result to be available to the physician or delay in scheduling the patient's RBC transfusion visit. Also, $33.6 \%$ of patients traveled 25 to $50 \mathrm{~min}$ between the transfusion center and their residence during each day of the RBC transfusion visit. Traveling such distance can be very inconvenient for the patient, especially if they rely on public transportation, and can be a source of stress which certainly contributes to patient burden [12] Such results are important to assess the patient burden because when all activities are taken into account and considering that $14.0 \%$ of patients had more than five transfusions over a 1-year period in our study, these times spent by the patients in relation to RBC transfusion are considerable and can certainly have an impact on the patient's quality of life. 
We should also consider that this time burden is in addition to the time burden of chemotherapy treatment that those patients with cancer must undergo, which was estimated to $5.7 \pm 6.9 \mathrm{~h}$ in a published retrospective survey study [13].

This study has several potential limitations and risks, associated with conducting chart review studies, such as abstraction errors, no control over time reported in the chart, missing data, and variation in abstraction methods. These were addressed in this study by pre-screening of study sites to ensure that patient medical records maintained at the site contain the requisite data for completion of the forms, thorough training of the study site staff on the protocol, CRF and EDC data entry procedures, use of one study staff person to perform the data abstraction at each site, programming of validation rules and edit checks in the EDC system, and regular monitoring of data entered to ensure data quality and adequate sample size to account for the possibility of missing data.

\section{Conclusion}

In conclusion, this study provides data on the patient time burden associated with RBC transfusion treatment. CIA patients experience substantial time burden when being treated with RBC transfusion in addition to the burden already compromised by chemotherapy.

Acknowledgments The authors would like to thank Elizabeth Donahue, Stephanie May, Noreen Lordan, Marilyn Stolar, Ray Shieh, Sunning Tao, and all the participating centers for their contribution to this study.

Conflict of interest Authors Marie-Pierre Desrosiers and Krista Payne are employees of United BioSource Corporation, a consultancy that has also received grants for other, unrelated research from Amgen Inc. Sanatan Shreay and Patricia Corey-Lisle are employed by Amgen Inc. and owns stocks.

Open Access This article is distributed under the terms of the Creative Commons Attribution Noncommercial License which permits any noncommercial use, distribution, and reproduction in any medium, provided the original author(s) and the source are credited.

\section{References}

1. Barrett-Lee P, Bokemeyer C, Gascon P et al (2005) Management of cancer-related anemia in patients with breast or gynecologic cancer: new insights based on results from the European Cancer Anemia Survey. Oncologist 10:743-757

2. Hayes JM (2007) Chemotherapy-induced anemia: treating it as a disease, not just a condition. Adv Stud Pharm 4(5):125-132

3. Rodgers GM (2008) Managing patients with chemotherapyinduced anemia. Adv Stud Med 8(10):346-351

4. Schwartz RN (2007) Anemia in patients with cancer: incidence, causes, impact, management, and use of treatment guidelines and protocols. Am J Health Syst Pharm 64(3 Suppl 2):S5-S13, quiz S28-30

5. Groopman JE, Itri LM (1999) Chemotherapy-induced anemia in adults: incidence and treatment. J Natl Cancer Inst 91 (19):1616-1634, Review. Erratum in: J Natl Cancer Inst 2000 Mar 15;92(6):497

6. Houts AC, Loh GA, Fortner BV et al (2006) Patient and caregiver time burden associated with anaemia treatment in different patient populations. Support Cancer Care 14:1195-1204

7. Meehan KR, Tchekmedyian NS, Smith RE et al (2006) Resource utilisation and time commitment associated with correction of anaemia in cancer patients using epoetin alfa. Clin Drug Invest 26(10):593-601

8. Minuk L, Chin-Yee I, Hibbert A et al (2008) Red blood cell transfusion and chemotherapy administration: a study of resource utilization. Common Oncol 5:598-603

9. Ueno W, Beveridge RA, Kales AN (2006) Costs of outpatient red blood cell transfusions. J Support Oncol 4(10):494-495

10. Declaration of Helsinki (2000) Adopted by the 18th World Medical Assembly General Assembly, Helsinki, Finland, 1964; amended by the 29th WMA General Assembly in Tokyo, Japan in 1975, the 35th WMA General Assembly in Venice, Italy in 1983, the 41st WMA General Assembly in Hong Kong in 1989, the 48th WMA General Assembly in Somerset West, Republic of South Africa in 1996, and the 52nd WMA General Assembly in Edinburgh, Scotland

11. MapQuest Software available at: http://www.mapquest.com/. Accessed March 17, 2012)

12. Payne S, Jarrett N, Jeffs D (2000) The impact of travel on cancer patients' experiences of treatment: a literature review. Eur J Cancer Care (Engl) 9(4):197-203

13. Fortner B, Tauer K, Zhu L et al (2004) The impact of medical visits for chemotherapy-induced anemia and neutropenia on the patient and caregiver: a national survey. Community Oncol $1: 211-217$ 